\title{
Baum des Jahres 2020: Die Robinie (Robinia pseudoacacia, Fabaceae), ein kontrovers diskutierter Neophyt
}

\author{
Veit Martin Dörken und Armin Jagel
}

\begin{abstract}
False acacia or black locust is the tree of the year 2020. It originates from North America, but is naturalised in many regions around the world. It provides robust wood and is a good bee pasture. As a heat and drought resistant robust species it could be a so-called climate change tree for the future. But it is also an invasive neophyte, which can suppress native vegetation, especially on poor soils.
\end{abstract}

\section{Zusammenfassung}

Die Gewöhnliche Robinie oder Falsche Akazie (Robinia pseudoacacia) ist Deutschlands Baum des Jahres 2020. Sie stammt aus Nord-Amerika, ist aber weltweit vielerorts eingebürgert. Sie liefert robustes Holz und ist eine gute Bienenweide. Als hitze- und trockenheitsresistente robuste Art könnte sie ein sogenannter Klimawandelbaum für die Zukunft sein. Die Robinie ist aber auch ein invasiver Neophyt, der vor allem an mageren Standorten die heimische Vegetation verdrängen kann.

\section{Einleitung}

Wie in den letzten 31 Jahren kürte das Kuratorium Baum des Jahres auch für 2020 wieder einen
Baum des Jahres. Dabei fiel die Wahl nicht auf eine bei uns ursprünglich heimische Baumart, sondern auf einen besonders im Naturschutz äu-

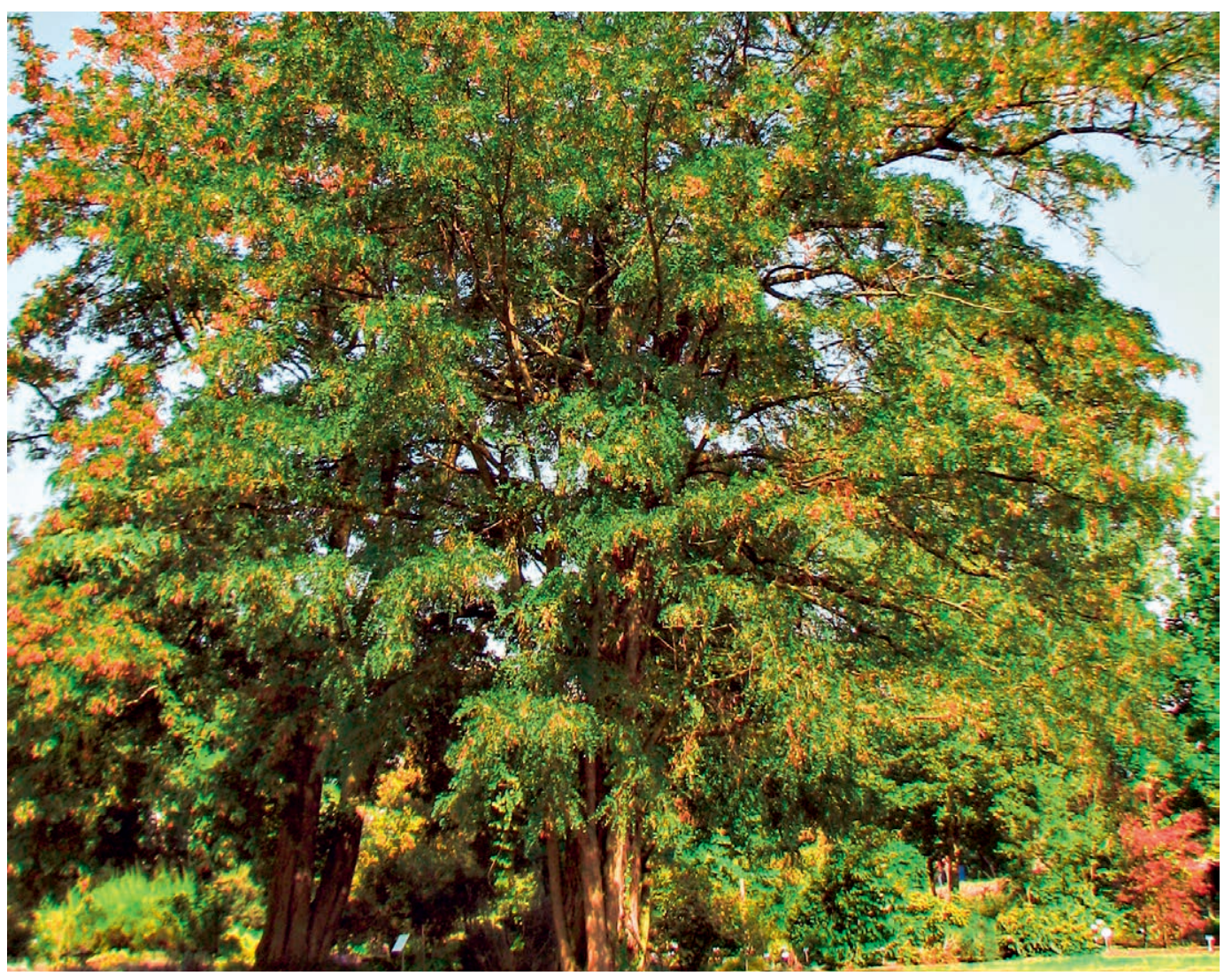

Abb. 1: Eine alte Robinie. (Foto: V. M. DörkeN) 
ßerst umstrittenen Neophyten, nämlich die aus Nordamerika stammende Robinie. Sie wurde bei uns als Zier- und Waldbaum um 1600 eingeführt (Sснӥтт et al. 2002). Verwilderungen der Robinie sind mittlerweile nicht nur auf Bahndämme und Industriebrachen beschränkt. Sie gedeiht auch in sonnig-warm exponierten Wäldern sowie auf Halbtrockenrasen und in Dünen. Durch ihre starke Ausbreitungstendenz verdrängt sie heimische Arten. Anderseits ist die Robinie bei uns eine der wichtigsten Bienentrachtpflanzen, der so genannte Akazienhonig erfreut sich großer Beliebtheit.

\section{Verwandtschaft}

Die Gewöhnliche Robinie (Robinia pseudoacacia) ist ein Vertreter der Fabaceae (Schmetterlingsblütler). Sie wird bei uns meist nur kurz als Robinie bezeichnet. Umgangssprachlich wird sie auch als Akazie bezeichnet. Die Gattung Acacia ist aber innerhalb der Fabaceae nicht näher mit Robinia verwandt. Die Gattung Robinia umfasst rund zehn Baum- und Straucharten. Von diesen werden zwar gelegentlich auch andere Arten bei uns als Ziergehölze gepflanzt, aber nur Robinia pseudoacacia hat sich als Neophyt bei uns etabliert. Manchmal wird die Robinie mit dem Japanischen Schnurbaum (Sophora japonoca) verwechselt, der im Rhein-Main-Gebiet gelegentlich im öffentlichen Grün gepflanzt wird. Schnurbäume blühen aber deutlich später im Jahr als Robinien.

\section{Verbreitung}

Robinia-Arten sind neuweltlich verbreitet mit einem Areal von Nord-Amerika bis Mexiko. Robinia pseudoacacia weist in Nord-Amerika zwei Teilareale in den östlichen Appalachen und in der westlichen Ozark-Region auf und ist dabei Bestandteil von Laubwäldern ( (Сснӥтт et al. 2002). Sie ist eine Lichtbaumart und kommt auf trockenen bis mäßig feuchten, durchlässigen Böden mit einer sauren bis alkalischen Bodenreaktion vor. Aufgrund der ausgeprägten Hitze- und Trockenheitsverträglichkeit wächst die Robinie auch auf Sand- und Kiesböden (Kiermeier 1993).

Da die Robinie außer in Nord-Amerika auch in geeigneten Klimaten auf anderen Kontinenten als Zierbaum gepflanzt wird und auch als Forst- baum eingesetzt wird, ist sie mittlerweile weit über ihr natürliches Areal verbreitet. Sie ist eine der wenigen bei uns winterharten Baumarten der Schmetterlingsblütler. In Nordrhein-Westfalen wurde sie zu einer der Charakterarten von Industriewäldern und Sukzessionsflächen, wie z. B. Schotterflächen entlang von Bahndämmen. Eine Zeit lang war sie dort der bevorzugte Baum, wenn es um die Begrünung von Bergehalden ging. Denn wie für Schmetterlingsblütler üblich, lebt auch die Robinie in Symbiose mit Luftstickstofffixierenden Bakterien, die in den unterirdischen Wurzelknöllchen zu finden sind. Der aus der Luft gebundene Stickstoff wird in pflanzenverfügbare Formen überführt. Aufgrund dieser Symbiose ist die Robinie geeignet, selbst auf kargsten Standorten wie Abraumhalden Fuß zu fassen.

\section{Morphologie}

\subsection{Habitus}

Die Robinie ist ein sehr raschwüchsiger, winterkahler Baum, der 30 m Höhe erreicht. Der Stamm ist bei der Wildform relativ kurz und teilt sich schon früh aufgrund der starken Tendenz zur Zwieselbildung. Dabei spaltet sich der Leittrieb in zwei gleichstarke Seitenäste auf. Die lichte Krone ist locker und sparrig verzweigt. Im Alter neigen

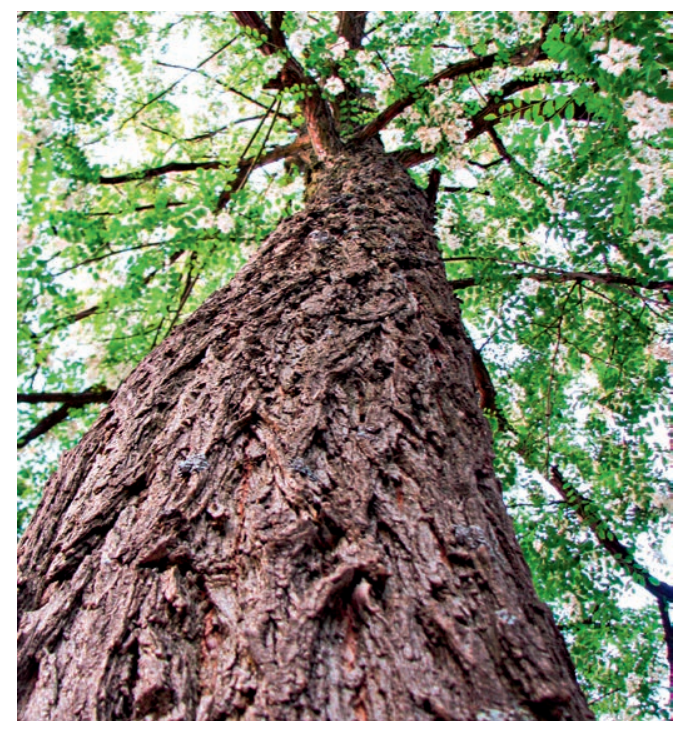

Abb. 2: Stamm einer Robinie mit rissiger Borke.

(Foto: V. M. DörkeN) 
Robinien zu Windbruch. Als der Orkan Kyrill im Januar 2007 über Deutschland wütete, war Nordrhein-Westfalen besonders stark betroffen. Damals wurden dort durch den Sturm viele Robinien, die als Straßenbäume gepflanzt waren, umgelegt.

Die Borke junger Triebe ist zunächst glatt, reißt aber mit zunehmendem Dickenwachstum längs auf. Der Stamm weist dann eine dicke, tief gefurchte graubraune Borke auf, die aus zahlreichen netzartig miteinander verbundenen Leisten besteht. Die Triebe von Robinia pseudoacacia sind im Unterschied zu denen der meisten anderen Robinien-Arten kahl und nicht klebrig. Es fehlt ihnen oft die Endknospe. Die seitlichen Achselknospen sind sehr unauffällig oder sie bleiben in den Blattnarben unter der Borke verborgen (Sснӥтт \& al. 2002, Roloff 2019).

An einer zunächst lang ausgebildeten Pfahlwurzel bilden sich zahlreiche Seitenwurzeln (Roloff 2019). Die Neigung zur Ausbildung von Wurzelbrut ist hoch und wird durch die Fällung des Baums stark gefördert. Aufgrund von Wurzelausscheidungen wird das Aufkommen von um Wasser und Nährstoffe konkurrierendem Unterwuchs aus anderen Arten verhindert (KIERMEIER 1993).

\subsection{Blätter}

Wie bei allen Schmetterlingsblütlern stehen bei der Robinie die Blätter wechselständig. Sie sind unpaarig gefiedert, bis etwa $30 \mathrm{~cm}$ lang und setzen sich aus 7-21 dünnen, annähernd gegenständigen Fiederblättchen zusammen. Diese sind in Form und Größe alle gleich gestaltet, deutlich gestielt, bis $5 \mathrm{~cm}$ lang und länglich-elliptisch. Die Spitze der Fiederblättchen ist leicht gebuchtet und hat eine kleine, oft unauffällige Grannenspitze.

Der Blattaustrieb erfolgt bei der Robinie relativ spät, zusammen mit dem der Esche (Fraxinus excelsior) erst Anfang Mai. Im Austrieb sind die Blattunterseiten leicht weißlich behaart, verkahlen jedoch rasch. Die Blätter haben an der Basis die zwei für Fabaceae typischen Nebenblätter, die hier aber nicht blattartig, sondern als etwa $2 \mathrm{~cm}$ lange Nebenblattdornen ausgebildet sind. Diese sind spitz und stechend und machen Gebüsche aus jungen Robinien ziemlich undurchdringlich. Die Blätter sind im Austrieb dunkel oliv- bis braungrün, über den Sommer dunkelgrün und werden nach einer wenig spektakulären gelben Herbstfärbung abgeworfen.

Die Fiederblättchen haben je nach Tageszeit eine bestimmte Stellung. In der Nacht richten sich die Blättchen in eine abwärts geneigte Schlafstellung, während sie am Tage wieder horizontal abspreizen. Je nach Lichtintensität können sich die Fiederblättchen auch bei zu hoher Sonneneinstrahlung schräg zum Einfallswinkel des Lichtes ausrichten. In Trockenphasen falten sich die Blätter nach oben zusammen, um die wasserabgebende Oberfläche zu reduzieren. Diese Bewegungen werden durch Gelenke an den Blattstielen ermöglicht.

\subsection{Blüten}

Robinien blühen sehr üppig mit oder nach dem Laubaustrieb von Mai bis Anfang Juni. Bereits junge Pflanzen können ab einem Alter von fünf Jahren die ersten Blüten bilden (Düll \& Kutzelnigg 2011). Die bis $30 \mathrm{~cm}$ langen Trauben setzen sich aus $10-30$ weißen, bis $3 \mathrm{~cm}$ großen Einzelblüten zusammen, die einen intensiven, weithin wahrnehmbaren Duft verströmen. Robinien werden von Bienen und Hummeln bestäubt. Die Blüten sind sehr nektarreich und produzieren täglich rund $2-3 \mathrm{mg}$ Nektar. Sie stellen damit bei uns die nektarreichste Trachtpflanze für Honigbienen dar (Düll \& Kutzelnigg 20II).

Die Blüten haben einen $1 \mathrm{~cm}$ langen Stiel, die fünf Kelchblätter sind miteinander glockig verwachsen. Die fünf Kronblätter zeigen die typische Gliederung in Fahne, Flügel und Schiffchen, wobei hier die beiden seitlichen Flügel von der median hinten stehenden Fahne überdeckt werden. Die Fahne hat einen auffälligen basalen, gelblichen Flecken. Der Fruchtknoten wird aus einem oberständigen Fruchtblatt gebildet, das zahlreiche Samenanlagen enthält. Die Blüten verfügen über einen Pumpmechanismus mit Bürsteneinrichtung, bei dem durch das Herunterdrücken des Schiffchens durch den Blütenbesucher zunächst die Narbe und dann nachfolgend die Pollenkörner an der Spitze des Schiffchens hervortreten. 


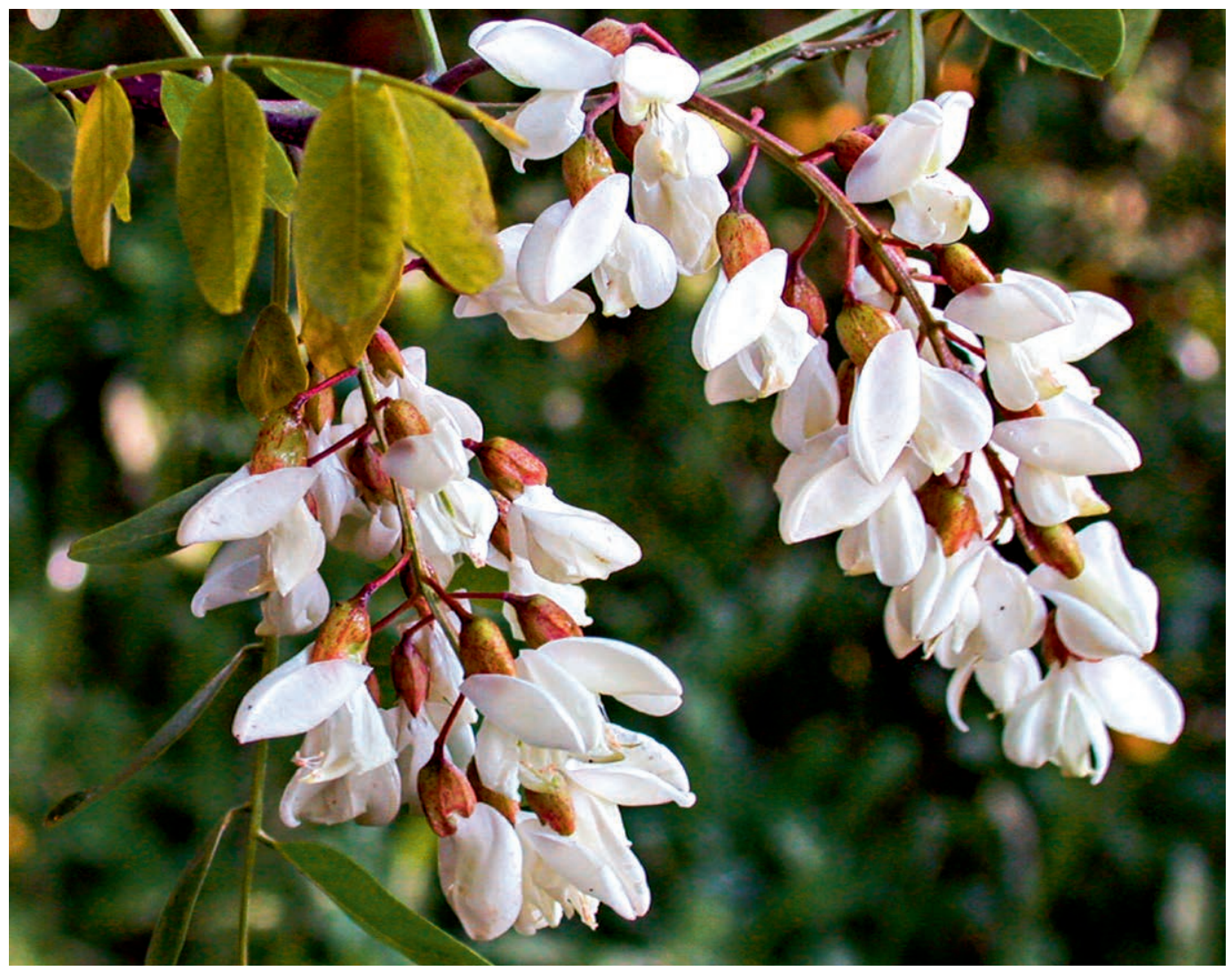

Abb. 3: Blütenstände der Robinie. (Foto: A. JAGEL)

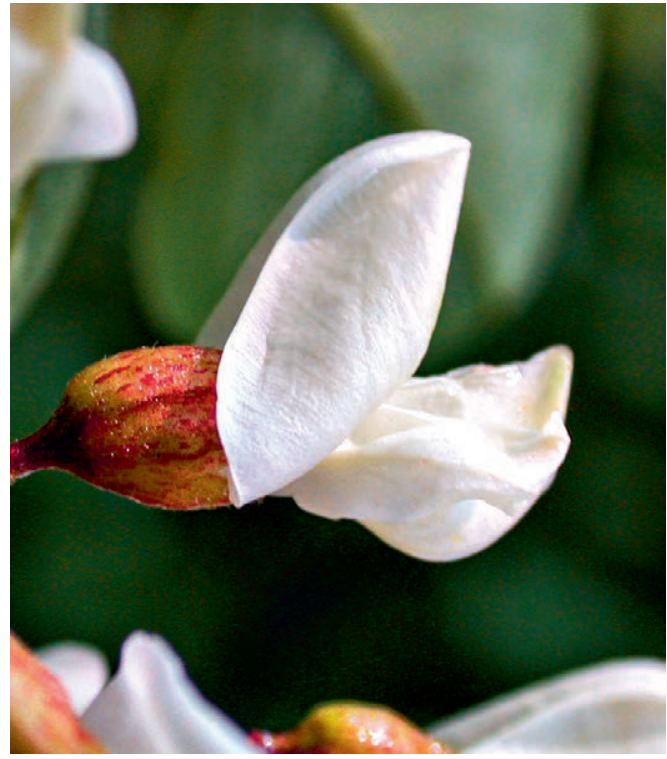

Abb. 4: Die Robinie hat eine typische Schmetterlingsblüte mit Fahne, Flügeln und Schiffchen. (Foto: A. JAGEL)

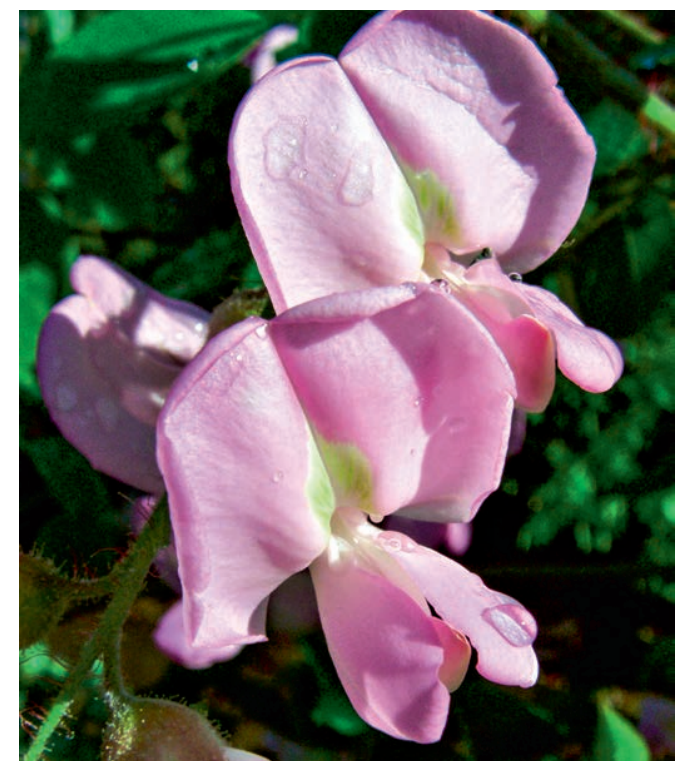

Abb. 12: Rosa Blüten von Robinia hispida.

(Foto: A. JAGEL) 


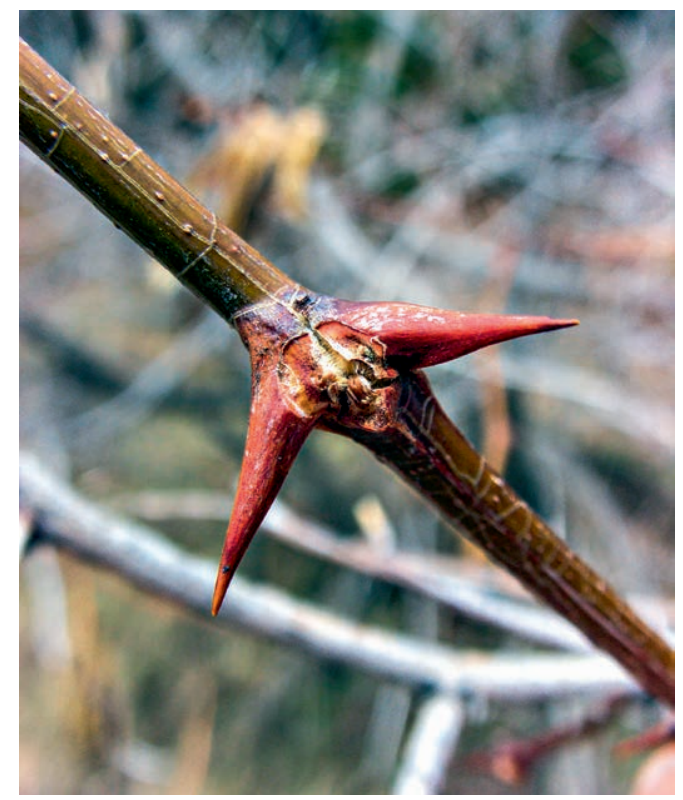

Abb. 5: Zweig mit Nebenblattdornen. (Foto: A. JAGEL)

\subsection{Früchte}

Die Frucht ist eine 5-12 cm lange, flache Hülse, die sich bei der Reife an Bauch- und Rückennaht öffnet, um die schwarzen, glänzenden Samen zu entlassen. Reife Früchte sind bräunlich, lederig und kahl. Das Nährgewebe ist sehr eiweißreich. Nach dem Freisetzen der Samen werden die Hülsen nicht unmittelbar abgeworfen, sondern bleiben bis zu einem Jahr eingetrocknet am Baum hängen.

\section{Verwilderungen und Naturschutz}

Robinien sind in Deutschland fast überall präsent. Robinien als Straßenbäume können sich aussamen und in der näheren Umgebung verwildern. Einbürgerungen erfolgen insbesondere an wärmeren Standorten im Flachland, z. B. in den Ballungsräumen an Rhein und Ruhr, deutlich weniger dagegen in den Mittelgebirgen. Besondere Aufmerksamkeit wird der Robinie bei der Rekultivierung von Industriebrachen geschenkt, wo sie Dominanzbestände ausbilden kann. So wurden auf dem Gelände der ehemaligen Zeche Zollverein Essen Robinien vor etwa 110-120 Jahren zur Haldenbefestigung gepflanzt. Diese Bestände gelten in NRW heute als die ältesten, die bereits ihr

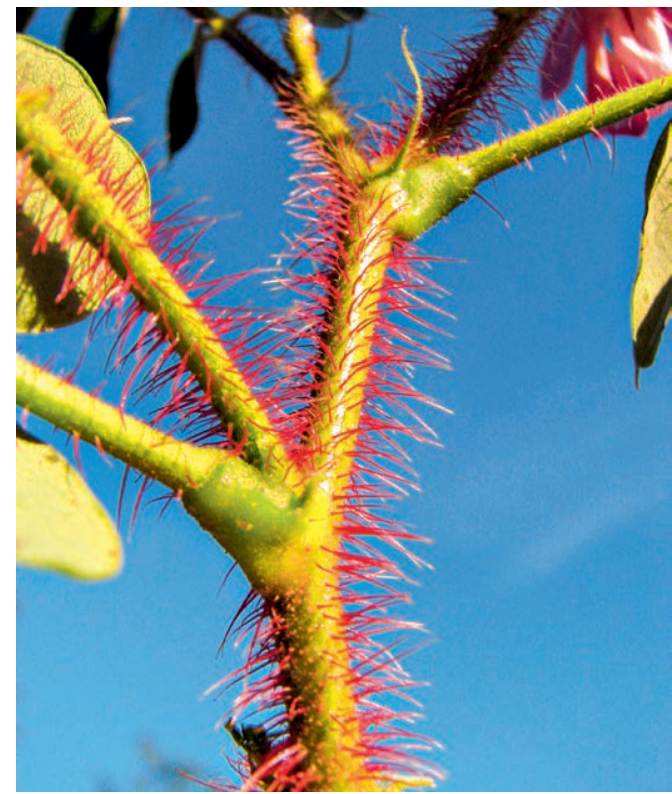

Abb. 11: Borstiger Trieb von Robinia hispida. (Foto: V. M. DörkeN)

Altersstadium erreicht haben und nun zunehmend zusammenbrechen (LANUv 2010).

Aufgrund ihrer ausgesprochenen Verträglichkeit gegenüber Hitze und Trockenheit ist die Robinie bei uns auch in vielen sensiblen trockeneren Ökosystemen wie Halbtrockenrasen und Sand-

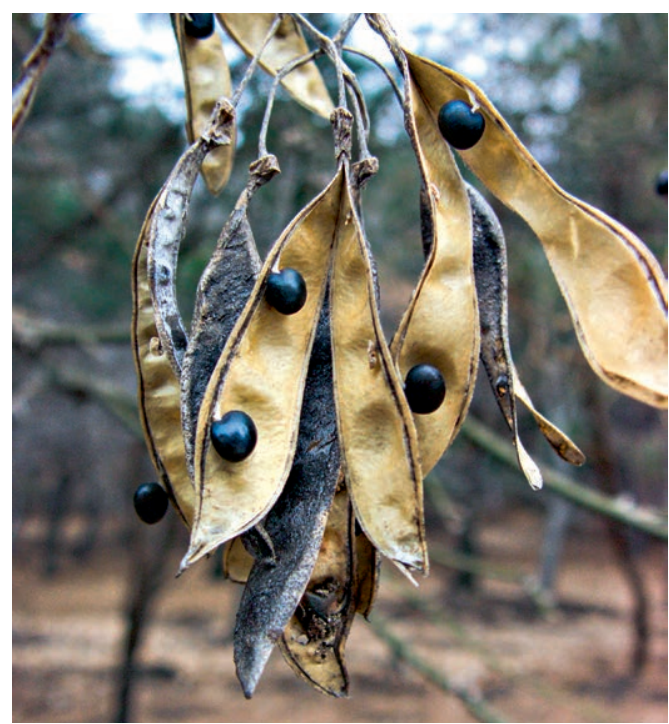

Abb. 6: Geöffnete Hülsen mit Samen. (Foto: A. JaGeL) 


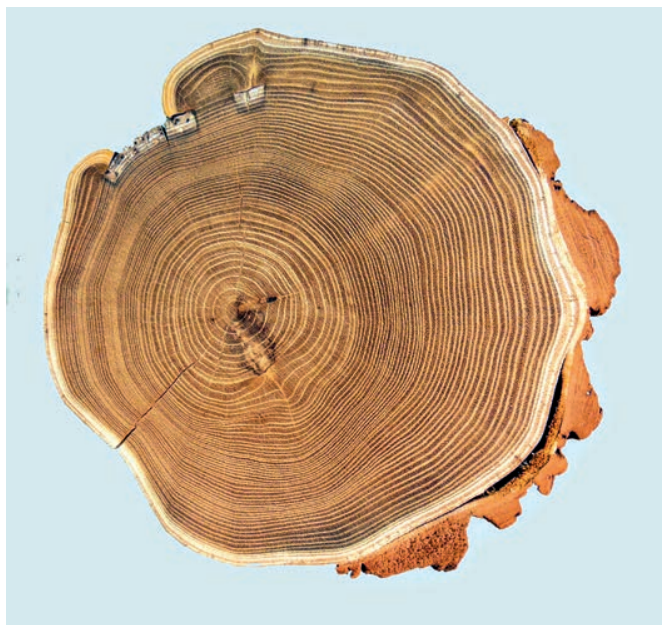

Abb. 7: Querschnitt durch das Holz der Robinie. (Foto: V. M. Dörken)

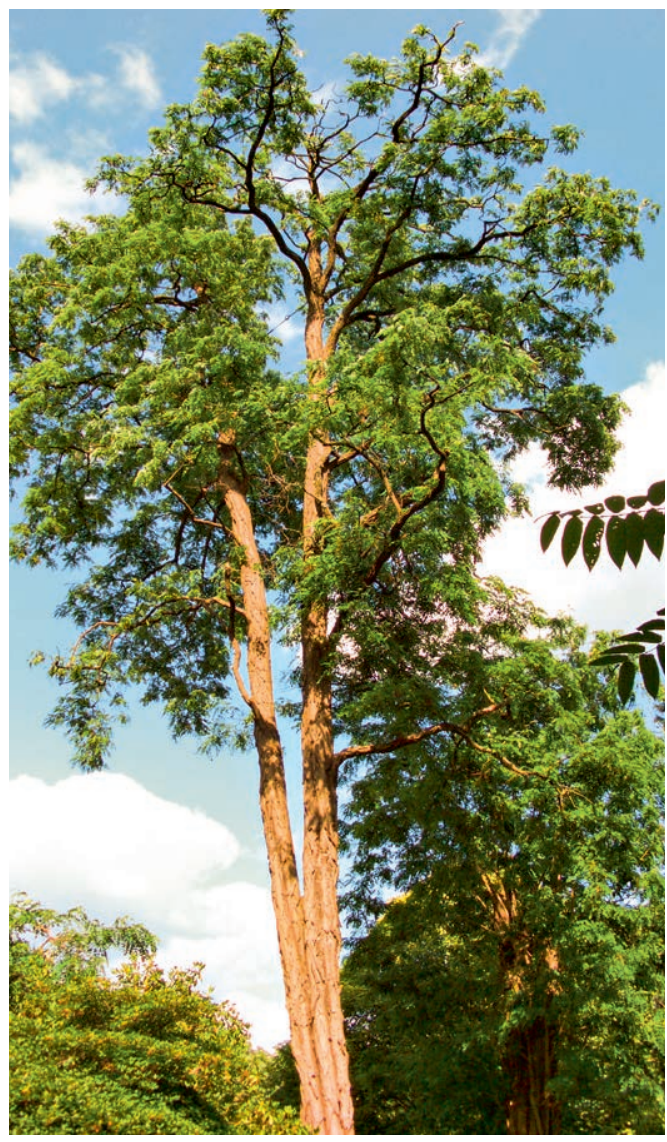

Abb. 8: Robinien verzweigen sich früh, so dass keine hohen geraden Stämme gebildet werden, die für eine Nutzung des Holzes bevorzugt werden. (Foto: A. JAGEL) magerrasen sowie in Wäldern zu finden, wo sie sich massiv ausbreiten kann und zur Verdrängung ursprünglich heimischer Arten führt. Daher wird die Robinie im Naturschutz oft als Problemneophyt angesehen.

Robinienblätter sind stickstoffreich und durch die Zersetzung der Laubstreu kommt es zu einer Stickstoffanreicherung im Boden, die zu einer nachhaltigen Veränderung der Artenzusammensetzung in der Krautschicht führen kann, bis hin zur Ausbildung von nitrophilen Pflanzengesellschaften (Schütt \& al. 2002, Düll \& KutzelNIGG 2011). Bekämpfungen durch Fällen oder Abbrennen führen in der Regel wegen der starken Regenerationsfähigkeit der Robinien nicht zum Erfolg. Einfaches Zurückschneiden führt daher nicht zum Absterben des Baums, sondern zu einem vermehrten Austrieb der Wurzelbrut und zur Verbuschung einer Fläche. Daher werden Stämme geringelt (ringförmiges Entfernen der Borke am Stamm), wodurch aber oft ebenfalls der Wurzelaustrieb gefördert wird. Bessere Erfolge können erzielt werden, wenn die Ringelung nicht komplett um den Stamm erfolgt, sondern nur teilweise und dadurch der Baum langsam geschwächt wird.

\section{Verwendung von Robinien}

Das Holz von Robinia pseudoacacia ist recht wertvoll. Es zeichnet sich durch seine Härte, Elastizität, Dauerhaftigkeit, schwere Spaltbarkeit und Tragfähigkeit aus. Robinienholz wird besonders als Konstruktions-, Gruben-, Bau- und Werkholz, aber auch zur Schwellen- und Werkzeugherstellung genutzt. Sowohl in der Bautischlerei als auch in der Drechslerei und Möbelschreinerei findet es Einsatz. Zudem weist Robinienholz einen hohen Brennwert auf (SсHÜTt \& al. 2002, Lieberei \& Reisdorff 2007). Nachteilig ist, dass viele Robinien keinen geraden, gleichmäßig gewachsenen Stamm mit erst hoch ansetzender Krone ausbilden. Wegen seiner Festigkeit kann das Holz kaum genagelt werden, stattdessen muss es aufwändig gebohrt und geschraubt werden. Sollen die sehr wetterfesten Bretter als Zaunlatten für Viehweiden genutzt werden, muss unbedingt die giftige Rinde entfernt werden. 


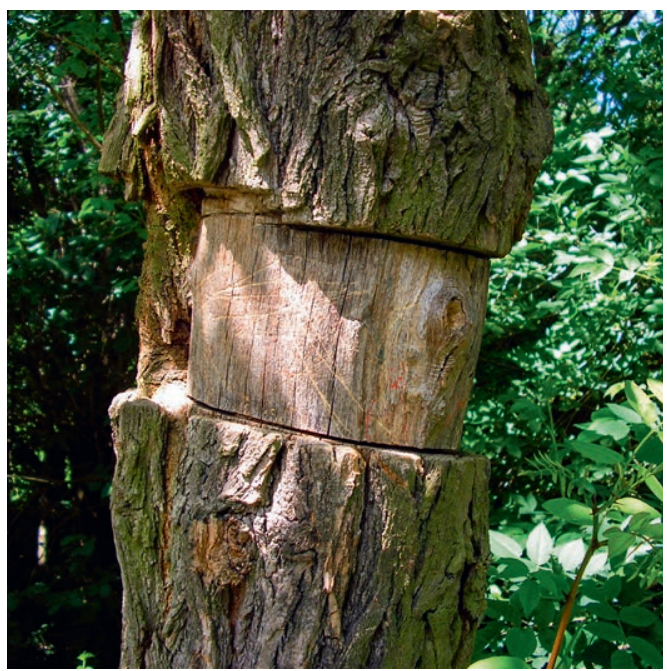

Abb. 9: Robinien gelten als invasiv und werden mancherorts bekämpft, indem ihre Stämme geringelt werden.

(Foto: P. GAusmanN)

Aufgrund des sehr schnellen Wachstums und der damit verbundenen hohen Biomasseproduktion wird Robinia neuerdings auch in Kurzumtriebs-Plantagen zur Gewinnung von Energiehholz kultiviert (Roloff 2019). Im Zuge des Klimawandels wird im Zusammenhang mit der Robinie auch immer wieder diskutiert, ob sie, obwohl sie stellenweise ein invasiver Neophyt ist, sich als so genannter Klimawandelbaum für bestimmte Zwecke eignet.

Wege ihrer Hitze- und Trockenresistenz sowie Unempfindlichkeit gegenüber Streusalzen und Abgasen wird die Robinie als Straßenbaum gepriesen. Diese Beliebtheit äußert sich auch darin, dass es in vielen Orten Straßen mit der Bezeichnung Akazienstraße gibt, so beispielsweise auch in Frankfurt und Dortmund. Als Straßenbaum findet häufiger die Sorte 'Bessoniana' Einsatz, die eine dichtere, kegelförmige bis ovale Krone sowie einen durchgehenden Leittrieb aufweist. Aufgrund des hohen Zierwertes wird Robinia pseudoacacia aber auch in großen Gärten und Parkanlagen gepflanzt. In kleineren Gärten und Straßenräumen findet meist die als Hochstamm veredelte Sorte 'Umbraculifera' Einsatz, die im Gegensatz zur Stammform eine kugel- bis eiförmige, dicht verzweigte Krone aufweist. Zudem ist diese Sorte blüten- und

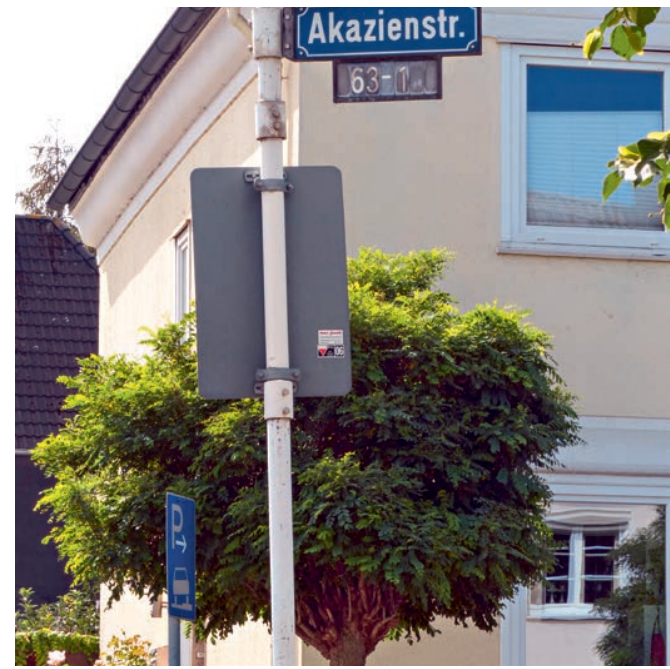

Abb. 10: Akazienstraße in Dortmund. Als Straßenbäume sind hier Robinien gepflanzt, die jedes Jahr zurückgeschnitten werden. (Foto: G. STEInEcke)

dornenlos. Robinien mit kugelig geschnittenen Kronen passen zudem gut in formale Gärten. Im Palmengarten stehen zwei kleinere Robinien vor dem Haus Leonhardsbrunn.

Auch die Sorte 'Frisia' (Gold-Akazie) zählt zu den gut geeigneten Stadtbäumen, die sich durch die gelblich grüne Farbe der Blätter auszeichnet. Sie hat mittlerweile die früher häufiger gepflanzte, ähnliche Sorte 'Aurea' abgelöst.

Wer eine Robinie in den Garten pflanzt, sollte achtsam sein, vor allem, wenn auch Kinder sich im Garten aufhalten. Denn für den Menschen sind Rinde, Blätter und Samen stark giftig. Die Rinde enthält Robin, Phasin, Syringin und Protocatechingerbstoffe, die Blätter Indican, Asparagin, ätherische Öle sowie Acactin. Die eiweißreichen Samen enthalten unter anderem Toxalbumine, Lectine sowie Glykoside (Roth \& al. 1994, DüLL \& Kutzelnigg 2011).

Neben der „Gewöhnlichen“ Robinie (Robinia pseudoacacia) wird sehr viel seltener noch die Borstige Robinie ( $R$. hispida) gepflanzt. Sie zeichnet sich durch einen grazilen und kleineren Wuchs sowie einen sehr auffälligen, exotisch anmutenden rosafarbenen Blütenflor aus. 


\section{Dank}

Für die Bereitstellung von Fotos bedanken wir uns herzlich bei Peter Gausmann (Herne), Gudrun Steinecke (Dortmund) und Hilke Steinecke (Frankfurt).

\section{Literatur}

Düll, R. \& Kutzelnigg, H. 2011: Taschenlexikon der Pflanzen Deutschlands und angrenzender Länder, 7. Aufl. Wiebelsheim.

Kiermeier, P. 1993: BdB-Handbuch, Teil 1. Laubgehölze. Fördergesellschaft „Grün ist Leben“. - Pinneberg.

LANUV NRw 2020: Robinie (Robinia pseudoacacia). - https:// neobiota.naturschutzinformationen-nrw.de/site/nav3/ArtInfo.aspx?ART =Pflanzen \&ID=d83aece3-8274-4cef-9ed6111e1114ce01\&MENU=Kurzbeschreibung
Lieberei, R. \& Reisdorff, C. 2007: Nutzplanzenkunde, 7. Aufl.- Stuttgart, New York.

Roloff, A. 2019: Baum des Jahres 2020: Die Robinie (Robinia pseudoacacia). - Ginkgoblätter 159: 23-26.

Roth, L., Daunderer, M. \& Kormann, K. 1994: Giftpflanzen - Pflanzengifte. - Landsberg.

Schütт, P., Sснuck, H. J. \& Sтімm, B. 2002: Lexikon der Baum- und Straucharten. - Hamburg.

\section{Anschrift der Autoren}

PD Dr. Veit Martin DöRken, Universität Konstanz, Fachbereich Biologie, Universitätsstraße 10, 78457 Konstanz, E-Mail: veit.doerken@uni-konstanz.de

Dr. Armin Jagel, Danziger Straße 2, 44789 Bochum, E-Mail: armin@jagel.nrw

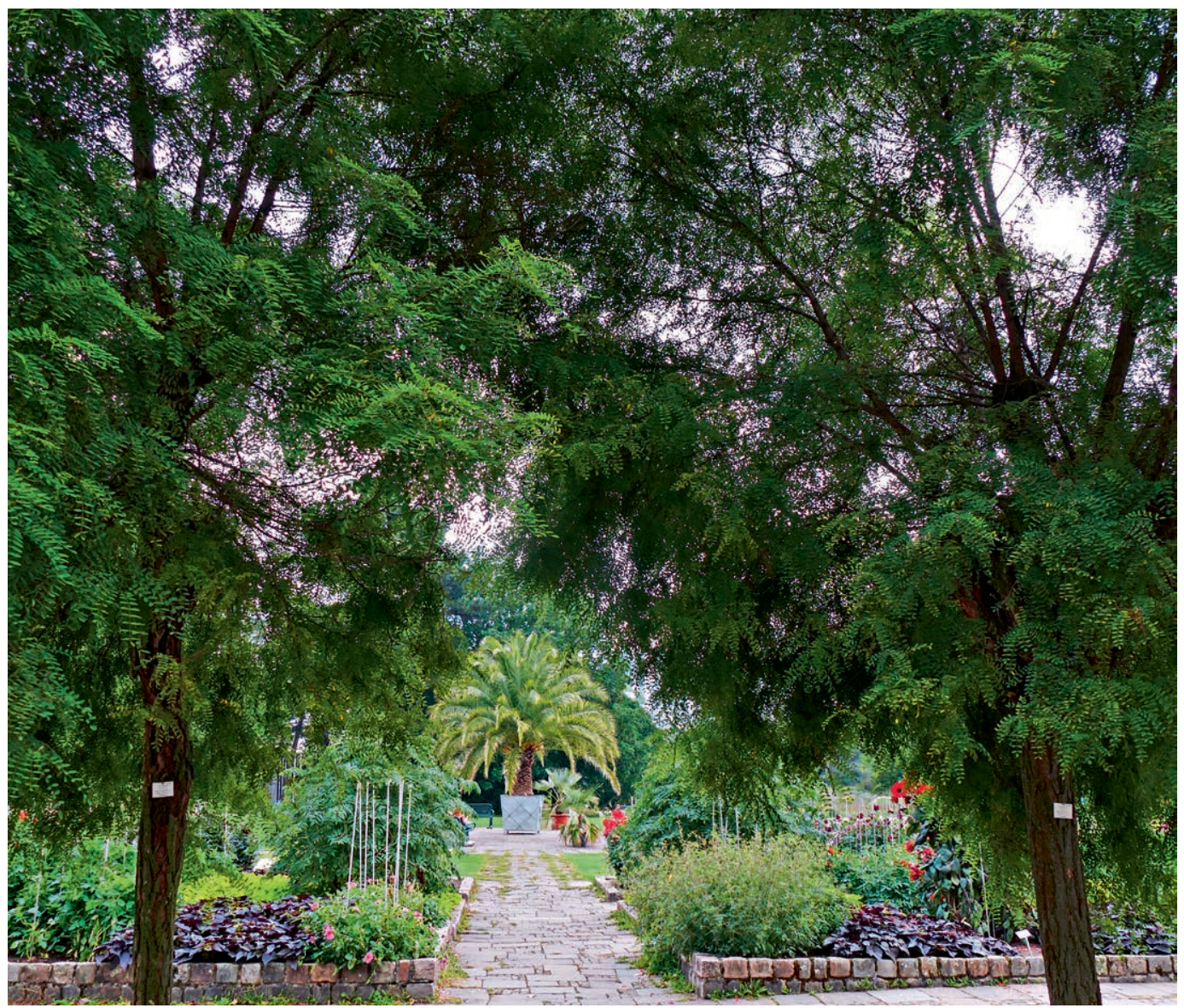

Abb. 13: Robinia pseudoacacia 'Mimosifolia', eine Sorte mit besonders feinem Laub, vor dem Haus Leonhardsbrunn im Palmengarten. (Foto: H. STEINECKe) 